\title{
Conf-9407/66--1
}

UCRL-JC-119454

PREPRINT

\section{Force Models for Particle-Dynamics Simulations of Grandular Materials}

\author{
Otis R. Walton
}

This paper was prepared for submittal to the

North American Treaty Organization

Advanced Study Institute for Mobile Particulate Systems

July 3-15, 1994

Cargese, Corsica

\section{December 1994}

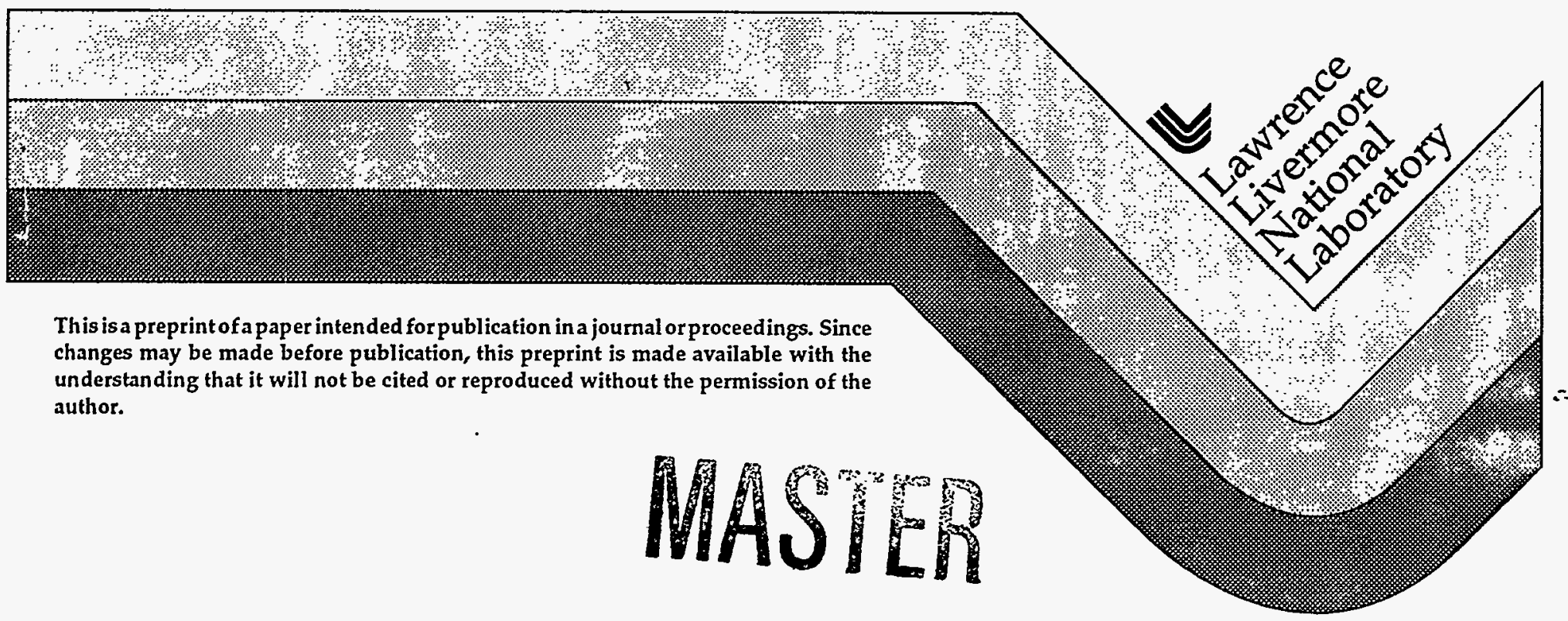




\section{DISCLAIMER}

This document was prepared as an account of work sponsored by an agency of the United States Government. Neither the United States Government nor the University of California nor any of their employees, makes any warranty, express or implied, or assumes any legal liability or responsibility for the accuracy, completeness, or usefulness of any information, apparatus, product, or process disclosed, or represents that its use would not infringe privately owned rights. Reference herein to any specific commercial product, process, or service by trade name, trademark, manufacturer, or otherwise, does not necessarily constihute or imply its endorsement, recommendation, or favoring by the United States Government or the University of Califomia. The views and opinions of authors expressed herein do not necessarily state or reflect those of the United States Government or the University of California, and shall not be used for advertising or product endorsement purposes. 


\section{DISCLAIMER}

Portions of this document may be illegible in electronic image products. Images are produced from the best available original document. 


-




\title{
FORCE MODELS FOR PARTICLE-DYNAMICS SIMULATIONS OF GRANULAR MATERIALS
}

\author{
Otis R. Walton* \\ University of California Lawrence Livermore National Laboratory \\ Livermore CA 94550 USA
}

\begin{abstract}
Engineering-mechanics contact models are utilized to describe the inelastic, frictional interparticle forces acting in dry granular systems. Simple analyses based on one-dimensional chains are utilized to illustrate wave propagation phenomena in dense and dilute discrete particulates. The variation of restitution coefficient with impact velocity is illustrated for a variety of viscous and hysteretic normal force models. The effects of interparticle friction on material strength in discrete-particle simulations are much closer to measured values than are theories that do not allow particle rotations.

Keywords: friction, inelastic, contact force, particulate, granular material, discrete particle, simulation, molecular dynamics, rotation, sliding, repose.
\end{abstract}

\section{Introduction}

Numerical simulation of the flow behavior of assemblies of macroscopic particles can be accomplished by adding engineering-mechanics models for contact forces to the well established molecular-dynamics algorithms for integrating the equations of motion of large numbers of molecules. Textbooks such as Allen and Tildesley's Computer Simulation of Liquids (1) and Hockney and Eastwood's Computer Simulation Using Particles (2) thoroughly discuss the numerical methods, near-neighbor searching algorithms and the pros and cons of various numerical integration algorithms for molecular-scale simulations. The main features that distinguish granular materials from molecules are the size and shape of the particles and the loss of available kinetic energy that occurs when particles collide and/or slide against each other. This energy loss is essentially a mode-coupling effect, with the original kinetic energy of the bodies being transformed into vibrational kinetic energy of the molecules that constitute the material, or being 'expended' creating new surface area as fractures are created or particles break. Because the time scale of typical molecular motions is so much shorter than the time scale of gross motion of the macroscopic particles, any energy transferred to molecular 'heat' is effectively 'lost' from the system of particles and is irrecoverable as kinetic energy of gross motion of individual grains or particles (3).

- Work performed under the auspices of the U. S. Department of Energy by the Lawrence Livermore National Laboratory under Contract W-7405-Eng-48 


\section{One Dimensional Systems}

Before describing detailed models for frictional, inelastic contact forces acting between real 3-dimensional particles it is often instructive to consider much simpler one-dimensional chains of identical particles (i.e., hard or soft rods or spheres) restricted to move along a straight line. In a one-dimensional hard-rod gas (composed of perfectly elastic, equal mass, particles) the initial velocity distribution never changes. Colliding particles simply exchange velocities. Such a system lacks the Layapunov instability (and non-head-on collisions) that leads to irreversibility in 2-D and 3-D hard-sphere gases (4). Non-head-on collisions between discs or spheres (in higher dimensional systems) also lead to equipartition of energy in all available degrees of freedom. Despite its shortcomings, a one-dimensional system can be used to illustrate important phenomena involved in all systems of colliding particles. In particular, as discussed in more detail below, normal and tangential components of interaction forces are often treated as independent or nearly independent quantities. The resulting normal-direction interaction models of higher dimensional systems are identical to those used to describe the head-on collisions involved in one-dimensional systems.

\subsection{ELASTIC SPHERES}

Theory $(5 ; 6)$, experiment $(7)$, and dynamic finite-element model calculations (8) have all demonstrated that quasi-static non-linear 'springs' at the point of contact between two elastic spheres, colliding at relatively low velocities along the line joining their centers, can adequately model almost all of the pertinent physics of the resulting nearly-perfectly-elastic collisions. The main feature inadequately modeled by such an approach is the less than one percent of the initial kinetic energy that goes into internal and/or external acoustic waves 'rattling around' inside the spheres and constituting the 'noise' we hear transmitted through the air when they collide. Introduction of a small amount of hysteresis into the non-linear springs (either via viscous forces or as a strictly position dependent loss) can approximately account for the energy 'lost' to such effects. More importantly, theory $(9 ; 10)$, experiment $(7 ; 10)$, and dynamic finite-element calculations (8) show that for impacts with velocities greater than a relatively small threshold value, inelastic plastic work or surface damage results in substantial energy loss, the magnitude of which increases as the relative impact velocity increases. Mathematical models for such moderatelyor highly-inelastic contacts are discussed in subsequent sections of this paper.

Hertz solved the linear elasticity equations for elastic bodies in contact $(5 ; 6$; 10). The resulting quasistatic force displacement relation for displacements along the line of centers between two contacting, identical elastic spheres is given by

$$
F=\frac{1}{3} \frac{E}{\left(1-\nu^{2}\right)} \sqrt{2 R} \alpha^{3 / 2}
$$

where $E$ is Young's modulus, $\nu$ is Poisson's ratio, $R$ is the spheres' radius, and $\alpha$ is the relative displacement after initial contact, (i.e. the 'virtual' overlap the two original spherical shapes would have as the actual elastic spheres deform at the point of contact). 
A Hertzian contact model- (i.e. Eqn. 1) is appropriate only for particles that contact over a relatively small contact area (as is the case for spheres, or could also be the case where contact is primarily via a small number of asperities that each may individually be approximated as nearly spherical in nature). An important feature of Hertzian contacts between spheres, that differs from the behavior that would be exhibited by a system of one-dimensional rods, is the duration of time adjacent particles remain in contact during binary collisions. Two colliding identical length rods (assuming plane-strain solution to elastic wave equation for stress waves traveling through the material) will remain in contact for the time it takes an elastic wave to traverse the length of each rod and back (i.e., two sound transit times through a rod). Two spheres interacting via the non-linear Hertzian contact force, on the other hand, will remain in contact a time, $\tau$, given by (6),

$$
\tau=2.94\left(\frac{5 \sqrt{2} \pi \rho}{4} \frac{1-\nu^{2}}{E}\right)^{2 / 5} \frac{R}{v_{n}^{1 / 5}},
$$

where $\rho$ is the density of the spheres, $R$ is their radius and $v_{n}$ is the incident relative velocity in the normal direction. This contact time is typically tens to hundreds of sound transit times across one of the spheres (depending on the incident velocity). The validity of Eqn (2) has been established both experimentally (7) and through dynamic finite-element simulation of colliding spheres (8).

\subsection{WAVE PROPAGATION IN 1-D SYSTEMS}

Several important features of 'wave propagation' phenomena in granular media can be illustrated by considering idealized systems of identical particles restricted to move in one dimension. Many of the qualitative features of this behavior can be extrapolated to 'real' systems in higher dimensions.

Particles in Continuous Contact:

A one-dimensional chain of spheres (e.g., beads on a wire), held in contact with each other by an externally applied boundary load, behaves very much like a sequence of point masses connected by springs. For large disturbances the non-linear (i.e., Hertzian) nature of the springs needs to be taken into account; however, for small disturbances we can approximate the behavior with linear springs whose stiffnesses correspond to the slope of the Hertzian force-displacement curve (i.e., Eqn 1) under the confining load. If we let $u_{i-1}, u_{i}$, and $u_{i+1}$, represent the displacements from their equilibrium positions of three adjacent point masses in the 1-D chain, and let $\Delta x$ be the equilibrium spacing between adjacent masses, $K$ the stiffness of the springs between adjacent masses, and $m$ the mass at each point, then the force acting on the $i$ th particle, $F_{i}$, is given by

$$
F_{i}=K\left(u_{i-1}-u_{i}\right)-K\left(u_{i}-u_{i+1}\right) \text {. }
$$

Using Newton's 2nd Law (i.e., $F=m a$ ) and multiplying the right hand side of Eqn (3) by $\frac{\Delta x^{2}}{\Delta x^{2}}$ we obtain

$$
m \ddot{u}_{i}=K \Delta x^{2}\left(\frac{u_{i-1}-2 u_{i}+u_{i+1}}{\Delta x^{2}}\right) .
$$


If we identify the factor in parenthesis on the right hand side of Eqn (4) as a finite difference approximation to the second derivative of $u$ with respect to $x$, we obtain an approximate second order, ordinary differential equation,

$$
\frac{d^{2} u}{d t^{2}} \approx \frac{K}{m} \Delta x^{2}\left(\frac{d^{2} u}{d x^{2}}\right)
$$

Comparing this to the one dimensional wave equation,

$$
\frac{\partial^{2} u}{\partial t^{2}}=c^{2} \frac{\partial^{2} u}{\partial x^{2}}
$$

we can identify the sound speed, $c$, with the quantity $\sqrt{\frac{K}{m}} \Delta x$. For spheres of diameter, $\sigma$, the spacing between adjacent spheres in contact is just one diameter so that we have $\Delta x=\sigma$. Thus, we identify Eqn (5) with the propagation of small disturbances, and we see that they propagate at a velocity that is proportional to the particle size and increases with the square root of the effective stiffness acting between adjacent spheres at their contact point. For elastic spheres with Hertzian (Eqn 1) contacts then, we would expect the wave propagation speed to vary with the 1/6 power of the confining load. Extrapolating this one-dimensional wave propagation analysis to higher dimensional assemblies is relatively straight forward, with the major change being that a quantity $n K$ needs to be substituted for $K$, where $n$ is the effective number of 'springs' acting (per sphere) between adjacent layers of spheres (layers perpendicular to the propagation direction). More complex analyses (11) including both normal and tangential springs at contacts have been used to infer the variation of wave speed in sand with depth of burial or the bulk modulus of random assemblies of contacting spheres.

Rigid Particles not in Contact:

Another somewhat simplistic analysis can serve to illustrate the differences between systems that are vibrationally fluidized and those whose particles are experiencing continuous contact with their neighbors. In rapid flows or fluidized conditions, particles usually experience isolated binary collisions and do not experience continuous contacts. In highly energetic flows or vibrational situations the time between collisions can be of much longer duration than the contact time of a collision. If the scale separation is sufficiently large, the system can be modeled by rigid spheres that interact via instantaneous rigid-body collisions. In such a system 'information' or disturbances are propagated by the motion of the particles.

The most idealized system we can utilize to illustrate the mechanism of wave propagation is a stationary set of perfectly rigid spheres, equally spaced along the $\mathrm{x}$-axis. A particle striking one end of the chain at a velocity, $v_{o}$, will start a oneparticle long 'wave' propagating down the chain. If the individual collisions are perfectly elastic, the total kinetic energy is constant, so at any one time there will be one particle moving at a velocity, $v_{o}$. If the average gap between particles' surfaces is $\Delta s$, and the particle diameter is $\sigma$, we find the 'wave' travels a distance $\sigma+\Delta s$ in a time, $\tau=\frac{\Delta s}{v_{0}}$. Thus, the effective 'wave velocity', $c$, is

$$
c \approx \frac{\sigma+\Delta s}{\Delta s} v_{o}=\frac{v_{o}}{f_{v}}
$$


where, $f_{v}$ is the void fraction. Since this model assumes perfectly rigid particles, the propagation velocity diverges as the void fraction approaches zero.

Elastic Particles - Finite Contact Time, $\tau_{c}$

If we consider more realistic elastic particles in a one-dimensional geometry wherein they are initially at rest equally spaced along the constraining line, we can again examine the response to a single particle hitting the chain at one end. Elastic particles have a finite contact time, $r_{c}$ (e.g., see Eqn 2), during which the colliding particle decelerates and stops while the contacted particle accelerates. We can approximate the effect of this finite contact time on the propagation behavior by assuming that each of the two particles has an average velocity during the time of contact that is approximately half of the incident colliding particle's velocity. Then during the time the particles are in contact they will translate a distance, $\delta_{c}=\tau_{c} \frac{\nu_{0}}{2}$. If we want to consider only cases with isolated collisions we need to require that the interparticle gap width, $\Delta s$, be greater than $\delta_{c}$. Then, between contacts the particle traveling at a velocity, $v_{o}$, moves a distance, $\Delta s-\delta_{c}$, and the corresponding time of flight between contacts is, $\tau_{g}=\frac{\Delta_{s}-\delta_{c}}{v_{0}}=\frac{\Delta_{s}}{v_{0}}-\frac{\tau_{c}}{2}$. Thus the 'wave' travels a distance $\Delta s+\sigma$ in a time $\tau_{c}+\tau_{g}$. The resulting propagation velocity then becomes,

$$
c \approx \frac{(\sigma+\Delta s) v_{0}}{\Delta s+\frac{v_{0} \tau_{c}}{2}}
$$

Because of the second term in the denominator, this velocity is clearly slower than that given in Eqn (7) for rigid spheres with instantaneous collisions.

The above analysis is too simplistic to provide quantitative estimates of the velocity of propagation of a disturbance in a random vibrating assembly of inelastic spheres or disks. It does clearly show, however, that there are two factors that contribute to wave velocities in non-contacting granular assemblies. One related to the velocity of the particles in the system and the other depending on the duration of contact in collisions. Both factors need to be considered in estimating transit. times through vibrating or rapidly flowing beds of granular materials.

\subsection{INELASTIC SPHERES}

When two spheres of elastic-plastic material are pushed together the forcedisplacement only follows Hertz's solution, Eqn (1), until the onset of plastic yield, which occurs when the total applied load is approximately (10)

$$
F_{Y} \approx 21 R^{2} \frac{\left(2-\nu^{2}\right)^{2}}{E^{2}} Y_{o}^{3},
$$

where $Y_{o}$ is the yield strength of the material. Any additional load results in plastic deformation of the region of the contact and changes the loading to a nearly linear relation for any additional increment of the normal load $(9 ; 8)$.

The plasticly deformed region of the contact, upon subsequent unloading, will act somewhat like an elastically compressed rod of a diameter comparable to the contact spot diameter, or more precisely like an elastic sphere with a larger radius, and thus a 'stiffer' Hertzian unloading curve (9). 
A variety of mathematical models have been proposed to approximate the physical behavior of this normal direction inelastic behavior. The simplist is a damped harmonic oscillator, or spring-dashpot 'joint element' acting between the two bodies in contact. Such a model has been used in a variety of granular flow simulation models $(12 ; 13 ; 14)$. Oden and Martins (15) suggested several forms of damped, non-linear springs that could provide realistic energy losses during collisions. Their proposed force-displacement function was of a general form

$$
F=-K \alpha^{n}-D \alpha^{l} \dot{\alpha}^{m}
$$

where $K$ and $D$ are constants, the exponent $n$ could be 1 or $3 / 2$ for Hookean or Hertzian spring force, respectively, setting $l$ to zero and $m$ to one would produce linear damping, and the dot in the last term indicates the time derivative. They generally preferred values of $l$ equal or greater than one, and $m$ greater than $1 / 2$. Introduction of the position dependent weighting factor, $\alpha^{l}$, in the damping term allows a rich variety of behavior to be modeled with the relatively compact form for the normal force.

The simple damped harmonic oscillator (i.e., $n=1, l=0$, and $m=1$ in Eqn. 10) has a few advantages and some shortcomings. Such a linear model is very fast to calculate. It also produces a constant coefficient of restitution (i.e., ratio of recoil to incident normal-direction relative speed) independent of the incident velocity. A constant coefficient of restitution is often utilized in kinetic theory models of rapid granular flow behavior, so it can be useful in comparison with such theories. A constant coefficient of restitution, however, is not representative of the behavior exhibited by most real spheres in collisions. Instead, the coefficient of restitution usually decreases monotonically with increasing impact velocity as greater damage or plastic deformation takes place at higher impact speeds. Another drawback of a simple linear damping term is the discontinuous nature of the resulting forcedisplacement curve. The damping term is usually inactive (i.e., set to zero) unless the spheres are touching. Then it is discontinuously 'turned on' with its maximum value at contact (since the relative velocity is a maximum when the particles first make 'contact').

Selecting a position dependent weighting for any viscous term (i.e., selecting a non-zero value for $l$ in Eqn 10) eliminates the discontinuity when particles first establish contact. For example, if we consider the case where $n=3 / 2$ and $m=1$ in Eqn 10 (i.e., a 'damped' Hertzian spring) combined with various choices for $l$, we obtain a variety of behaviors for the resulting simulated collisions. For $l=$ 0 , or a constant damping term, we not only have a discontinuity in the normal force when particles first make contact, but, the effective coefficient of restitution increases as the relative approach velocity increases - contrary to experimental and finite element model results (see Figure 1). Setting $l=1 / 2$ allows the damping term to effectively scale with the slope of the Hertzian spring force curve and produces a constant coefficient of restitution, since the damping factor in this case is continuously adjusted to maintain a fixed fraction of 'critical damping' for each incremental step along the force-displacement loading/unloading path. Setting $l=1$ produces a velocity dependence of the coefficient of restitution that is very similar to that observed experimentally for impacts with metal spheres (7) (see Figure 1). 


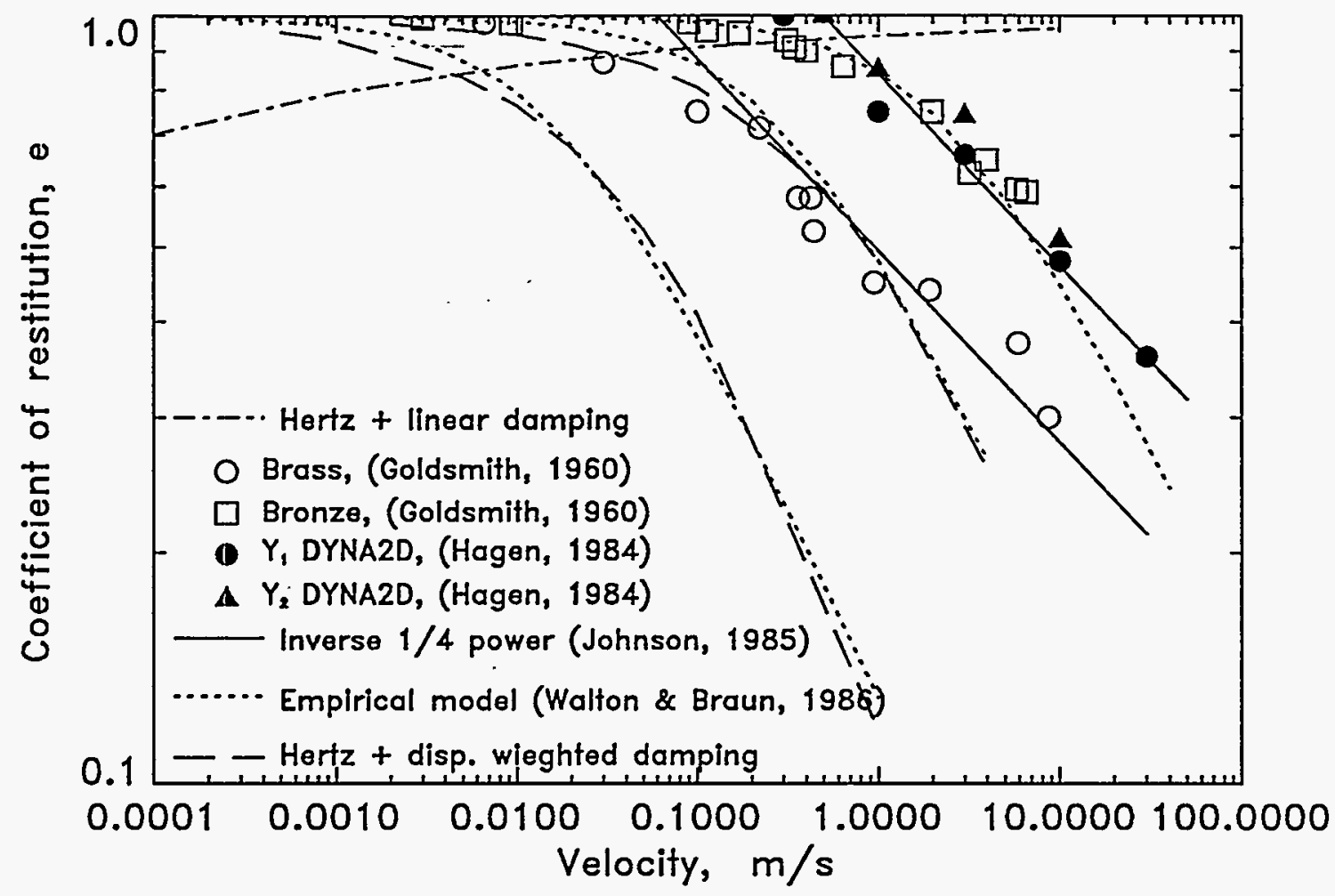

Fig. 1. Variation of coefficient of restitution with impact velocity, after Walton (8), for a variety of models and as measured for metal spheres (7). The filled symbols are results of dynamic finite element simulations with an elastic-perfectly-plastic material model (8)

Thornton (9) uses position dependent. hysteresis in the normal force model, chosen to mimic the behavior of elastic-plastic spheres in contact, and obtains a realistic . coefficient of restitution variation with impact velocity. Walton and Braun (16) utilize a linearized approximation to the curves of Thornton and achieve realistic restitution coefficient behavior over a limited range of incident velocities.

\section{Two Dimensional Systems}

When two and three dimensional systems are considered it is necessary to include the effects of friction and particle rotations. For assemblies of discs in 2-D, tangential friction becomes essentially a one-dimensional problem. The tangential displacement of the surface of a disc results from both the rotation and the translation of the disc. Before discussing specific friction models, it is useful to consider an idealized contact between an elastic disc and a planar surface, assuming that no sliding occurs (i.e., equivalent to having an infinite coefficient of friction acting at the contact). As we will see, such a system is still subject to energy losses as contacts are formed and 'broken' (even with everything described in terms of linear springs).

Most theoretical and numerical treatments of elastic or inelastic contacts assume 
that the local stress field (near the contact) in the normal direction is unaffected by tangential strains or tractions. While there are confounding effects of the two stress fields (see (18)) the simplifications allowed by treating the normal direction independently are usually deemed worth the slightly reduced accuracy. In our discussion we will assume that the normal direction stress and/or strain can be treated completely independent of the tangential force acting. We will illustrate, on the other hand, how the tangential force in intimately tied to the normal load (or displacement) and that the resulting confounding effects of micro-slip and 'breaking' of tangential springs as normal loads are removed can be important in determining the subsequent motion.

For the sake of illustration, consider a disc contacting a horizontal planar surface. We can model the contact as composed of two 'springs' tied to the points on the surfaces of the disc and the plane where initial contact is made, one spring acting in the normal direction and one in the tangential direction. We can obtain expressions for the equations of motion of the disc by considering the forces acting on it. Let $x$ be the horizontal (i.e., tangential) displacement of the c.g. of the disc from its initial contact position, and let $y$ be the same in the vertical (i.e., normal) direction. Further, let $F$ be force, $K$ be spring constant, $R$ be radius and $\theta$ be the angular orientation of the disc relative to its orientation when initial contact is established. Essentially we assume that these 'contact surface springs' are 'established' the moment the disc encounters the plane, and they will be eliminated whenever the disc loses contact with the plane. The forces in the vertical and horizontal directions are given by

$$
F_{y}=-K_{y} y \quad \text { and } \quad F_{x}=-K_{x}(x+R \theta) .
$$

The normal force does not contribute to the torque, $N_{\theta}$, acting on the disc

$$
N_{\theta}=R F_{x}=-R K_{x}(x+R \theta) \text {. }
$$

The translational and rotational accelerations, then, are

$$
\ddot{x}=\frac{F_{x}}{m}, \quad \ddot{y}=\frac{F_{y}}{m}, \quad \ddot{\theta}=\frac{N_{\theta}}{I_{o}} .
$$

Combining Eqns 11, 12 and 13 we obtain,

$$
\ddot{y}=-\frac{K_{y}}{m} y, \quad \text { and } \quad(\ddot{x}+R \ddot{\theta})=-\frac{K_{x}}{m}\left(1+\frac{m R^{2}}{I_{0}}\right)(x+R \theta),
$$

where $I_{o}$ is the moment of inertia, $m$ is mass, and the double dot implies the second derivative w.r.t. time. Both of the equations in (14) are simple harmonic oscillators. If we want to design this model system so that the normal direction spring will be back to its equilibrium position at the same time as the tangential spring then we must set $K_{y}=K_{x}\left(1+\frac{m R^{2}}{I_{0}}\right)$. For uniform density discs $I_{o}=\frac{1}{2} m R^{2}$, and for spheres $I_{o}=\frac{2}{5} m R^{2}$. Thus for a disc to have the same frequency in the normal direction as it does in the rotational direction we must have $K_{t}=\frac{1}{3} K_{n}$, and for spheres, $K_{t}=\frac{2}{7} K_{n}$. According to Mindlin (17), however, the ratio of tangential 
to normal stiffnesses for real elastic materials lies in the range $2 / 3 \leq K_{t} / K_{n} \leq 1-$ depending on Poisson's ratio of the material. This means that for most real contacts the frequency of the rotational motion will be higher than that of the normal direction. This, further, means that if the contacting sphere or disc is initially rotating slightly, it could experience a reversal (or even more than one reversal) in the direction of its rotation during the time it is in contact with the surface. Maw et al. (20) have studied such motions of discs in some detail and have demonstrated experimentally and calculationally that such reversals are quite possible (and/or probable). Similar behavior is observed for spheres (24).

Because the two frequencies of Eqns 14 are unlikely to be exactly the same, it is very likely that the tangential 'spring' will still be experiencing some finite displacement from its equilibrium position when the particle comes off of the surface via the normal-direction response. When that happens, the tangential spring would be 'broken' in the model we have described. In a 'real' contact, 'microslip' would dissipate energy as the normal load was removed while some finite tangential strain remained 'in the spring'. Mathematical models of the effects of microslip and friction can easily be made by allowing the 'effective' origin of the tangential spring to become an additional parameter whose value depends on the past (and present) normal and tangential displacements of the two contacting objects. In Mindlin's model for such contacts (17) he keeps track of every reversal point during the time two objects remain in contact. Most implementations for systems of large numbers of particles compromise and only retain a single tangential displacement history dependent quantity $(23 ; 12 ; 21)$ (Thornton maintains up to 4 levels of reversals in his implementation of Mindlin's model (19)). Time and space constraints do not allow a complete discussion of the details of more complex friction and tractive rolling models here.

\section{Three Dimensional Systems}

The situation in three-dimensions is similar to that in two, except that the direction of sliding can now be anywhere in a plane. Because the tangential friction force are a history-dependent force, there is a minimum of three values that must be stored for each contact (to carry over to the next time step). These are the three components of the current friction force vector or the tangential strain vector at each contact. Codes that utilize a linear loading curve up to the friction limit are able to minimize contact force storage requirements to only these three quantities $(12 ; 23)$. More complicated non-linear force-displacement curves that keep track of reversals in the direction of slip similar to Mindlin's (17) theory (e.g., Thornton (19); Walton (21)) require as many as 7 to 10 quantities be saved for each contact. In detailed engineering contact analyses of machined parts or railroad wheels, it may be necessary to utilize non-linear loading behavior and expend considerable calculational effort to determine the exact evolution of frictional forces as objects roll, slide and spin against each other (e.g., Kalker(25)); however, for simulating the

behavior of granular solids it is not clear that the more complex non-linear models; with memory of the turn-around points, produce substantially different behavior than the linear models would with slightly different effective stiffnesses or with 
an appropriately scaled velocity-dependent damping term. Additional sensitivity studies need to be performed to establish just how much detail is absolutely necessary to capture the pertinent physics of granular flows. Recent simulations have demonstrated that frictional forces and particle rotations are necessary in order to match the measured strength and angle of repose behavior for assemblies of spheres (22). Similarly, careful determination of input parameter values for 3-D simulation models have produced simulated flows that agree well with measured velocity and density profiles in rotating drums and on inclined chutes.

\section{Acknowledgements}

This work was supported by the US Department of Energy, Office of Fossil Energy, Advanced Research and Technology Development under the Solids Transport Program administered by Pittsburgh Energy Technology Center. That support is gratefully acknowledged.

\section{References}

[1] Allen, M.P. and D.J. Tildesley, Computer Simulation of Liquids Clarendon Press, Oxford, (1987).

[2] Hockney, R.W. and J.W. Eastwood, Computer Simulation Using Particles, McGraw Hill, NY (1991).

[3] Schofield, J. and I. Oppenheim, "The Hydrodynamics of Inelastic Granular Systems", Physica A 196 (1993) 209-240.

[4] Hoover, Wm. G., Computational Statistical Mechanics, Elsivier Sci. (1991).

[5] Hertz, H., J. Math (Crelles' J.), 92, (1881).

[6] Timoshenko, S.P. and J.N. Goodier, Theory of Elasticity, 3rd ed., McGraw-Hill, NY, (1970).

[7] Goldsmith, W., Impact, E. Arnold Pub., London, (1960).

[8] Walton, O.R., "Numerical Simulation of Inelastic Frictional Particle-Particle Interactions", in Particulate Two-Phase Flow, M.C. Roco, ed., Butterworth-Heinemann, Boston (1993) 884-911.

[9] Ning, Z. and C. Thornton, "Elastic-Plastic Impact of Fine Particles With a Surface", Powders 8 Grains 99, Thornton, ed., (1993) 33-38.

[10] Johnson, K.L., Contact Mechanics, Cambridge Univ. Press, Cambridge (1985).

[11] Duffy, J. and R.D. Mindlin J. App!. Mech. 24, (1957) 585. See also: Walton, K., "The Effective Elastic Moduli of a Random Packing of Spheres", J. Mech. Phys. Solids 85(2), 213-226 (1987).

[12] Cundall, P.A. and O.D.L. Strack, "A Discrete Numerical Model for Granular Assemblies", Geotechnique, 29, 47-65 (1979).

[13] Walton, O.R., "Explicit Particle Dynamics Model for Granular Materials," Numerical Methodo in Geomechanics Edmonton 1982, Z. Eisenstein, ed., 1261-1268, (1982).

[14] Pöschel, T. and H.J. Herrmann, "Size Segregation and Convection," HLRZ preprint 28/94, Jülich, Germany (1994).

[15] Oden, J.T. and J.A.C. Martins, "Models and Computational Methods for Dynamic Friction Phenomena," Proceedings, Fenomech III in: Computer Methods in Applied Mecharics and Engineering, North Holland, Amsterdam (1984).

[16] Walton, O.R. and R.I. Braun, "Viscosity and Temperature Calculations for Assemblies of Inelastic Frictional Disks, ${ }^{\prime}$ J. Rheology, 80(5), 949-980 (1986).

(17) Mindlin, R.D., J. Appl. Mech. (Trans. ASME) 16,-259 (1949).

[18] Walton, K. "The Oblique Compression of Two Elastic Spheres," J. Mech. Phys. Solids, 26, 139-150 (1978).

[19] Thornton, C. and C.W. Randall "Applications of Theoretical Contact Mechanics to Solid Particulate System Simulation," Micromechanics of Granular Materials, M. Saake and J.T. Jenkins, eds., Elsevier Sci. Pub., Amsterdam (1988) 133-142.

[20] Maw, N., J.R. Barber and J.N. Fawcett "The Role of Elastic Tangential Compliance in Oblique Impacts," Trans. ASME, J. Lub. Tech., 20, 327-344 (1981). 
[21] Walton, O.R., "Numerical Simulation of Inclined Chute Flows of Monodisperse, Inelastic, Frictional Spheres," Mech. of Mat. 16 (1993) 239-247.

[22] Walton, O.R. "Effects of Interparticle Friction and Particle Shape on Dynamic Angles of Repose via Particle-Dynamics Simulation," in Proceedings of Workshop on Mechanics and Statistical Physics of Particulate Materials, J. Jenkins and J. Goddard, eds., LaJolla, California, June 8-10, 1994.

[23] Hofstetter, K., (private communication, Caterpillar Inc., Peoria, IL, 1994)

[24] Foerster, S.F., M.Y. Louge, H. Chang, and K. Allia, Measurements of the Collisions Properties of Small Spheres," Phys. Fruids 6(3), 1108-1115 (1994).

[25] Kalker, J.J. Three-Dimenvional Elastic Bodies in Rolling Contact, Kluwer Academic Pub., Dordrecht, (1990). 